\title{
Genome-wide transcriptional regulation of estrogen receptor targets in fallopian tube cells and the role of selective estrogen receptor modulators
}

\author{
Georgette Moyle-Heyrman ${ }^{1,2}$, Matthew J. Schipma ${ }^{3}$, Matthew Dean ${ }^{1}$, David A. Davis ${ }^{1}$ and Joanna E. Burdette ${ }^{1 *}$
}

\begin{abstract}
Background: The fallopian tube epithelium is one of the potential sources of high-grade serous ovarian cancer (HGSC). The use of estrogen only hormone replacement therapy increases ovarian cancer (OVCA) risk. Despite estrogen's influence in OVCA, selective estrogen receptor modulators (SERMs) typically demonstrate only a $20 \%$ response rate. This low response could be due to a variety of factors including the loss of estrogen receptor signaling or the role of estrogen in different potential cell types of origin. The response of fallopian tube epithelium to SERMs is not known, and would be useful when determining therapeutic options for tumors arising from this cell type, such as HGSC.

Results: Using normal murine derived oviductal epithelial cells (mouse equivalent to the fallopian tube) estrogen receptor expression was confirmed and interaction with its ligand, estradiol, triggered mRNA and protein induction of progesterone receptor (PR). The SERMs 4-hydroxytamoxifen, raloxifene and desmethylarzoxifene, functioned as estrogen receptor antagonists in oviductal cells. Cellular proliferation and migration assays suggested that estradiol does not significantly impact cellular migration and increased proliferation. Further, using RNAseq, the oviduct specific transcriptional genes targets of ER when stimulated by estradiol and 4-hydroxytamoxifen signaling were determined and validated. The RNA-seq revealed enrichment in proliferation, anti-apoptosis, calcium signaling and steroid signaling processes. Finally, the ER and PR receptor status of a panel of HGSC cell lines was investigated including Kuramochi, OVSAHO, OVKATE, OVCAR3, and OVCAR4. OVSAHO demonstrated receptor expression and response, which highlights the need for additional models of ovarian cancer that are estrogen responsive.
\end{abstract}

Conclusions: Overall, the fallopian tube has specific gene targets of estrogen receptor and demonstrates a tissue specific response to SERMs consistent with antagonistic action.

Keywords: Estrogen, Fallopian tube epithelium, High-grade serous cancer, Ovarian cancer, Selective estrogen receptor modulators

\section{Background}

Ovarian cancer (OVCA) is the most lethal gynecological malignancy and fifth leading cause of cancer-related death in women [1]. The fallopian tube epithelium (referred to as oviduct epithelium in all species except primates) is one of the likely progenitor cell types for the

\footnotetext{
* Correspondence: joannab@uic.edu

'Department of Medicinal Chemistry and Pharmacognosy, University of Illinois at Chicago, Chicago, Illinois 60607, USA

Full list of author information is available at the end of the article
}

most common and deadly OVCA histotype, high-grade serous cancer (HGSC) with the alternative cellular source being the ovarian surface epithelium (OSE) [2]. Morphological, immunological, and gene expression analysis of HGSC tumors, suggest a close relationship to fallopian tube epithelium rather than OSE [3, 4]. Precursor lesions, termed the p53 signature, have been identified in the fimbriated end of the fallopian tube [5]. In cases of HGSC, these p53 signatures in the fallopian tube had the same mutation as their corresponding 
ovarian carcinoma [5]. Additional precursor lesions termed serous tubal intraepithelial carcinomas (STICs) have been identified in the fallopian tubes (but not ovaries) of women undergoing risk-reducing salpingooophorectomy, particularly in women with $B R C A 1 / 2$ mutations [6]. Furthermore, mouse models of HGSC have been produced by inducing BRCA, PTEN, and p53 mutation in the oviductal epithelium [7].

Risk factors for non-heritable OVCA include null parity, infertility, the number of lifetime ovulations and the use of estrogen only-hormone replacement therapy [8]. Estrogen is a steroid hormone that functions in multiple tissues in the body, including the fallopian tube epithelium. While the reproductive role of estrogen in the fallopian tube is to facilitate movement and maturation of eggs, sperm and fertilized embryos between the ovary and uterus [9], the function in terms of tumor initiation and progression is not clear. Estrogen signals in the cell through three main receptors. Estrogen receptor $\alpha(E R \alpha)$ and ER $\beta$ are ligand activated transcription factors [10]. $G$ protein coupled receptor (GPER) is a membrane bound factor that signals through a non-genomic mechanism [11]. Given the recent findings suggesting that OVCA is increased by hormone replacement therapy containing estrogen and the fallopian tube may be the source of HGSC, the estrogen receptor targets in the fallopian tube should be defined [12].

$E R \alpha$ and a gene target of estrogen signaling, progesterone receptor (PR), are prognostic biomarkers in OVCA [13]. ER $\alpha$ is expressed in $80 \%$ of HGSC, but PR is expressed in only $31 \%$ [13]. Successful treatment of OVCA with selective estrogen receptor modulators (SERM) therapy has been limited [14]. SERMs are ER ligands that function as either agonists or antagonists in a cell type specific manner [15]. Given that HGSC may arise from the fallopian tube, understanding the response of normal fallopian tube epithelium to estrogen and SERMS is important for understanding the implications of SERM therapy on OVCA risk.

Murine oviduct epithelial (MOE) cells were utilized to investigate estrogen signaling in a putative HGSC precursor. MOE cells are estrogen responsive and the SERMs 4-hydroxytamoxifen (4OHT), raloxifene (RAL) and desmethylarzoxifene (DMA) antagonize 17-ßestradiol $\left(E_{2}\right)$ in this cell type. The MOE specific transcriptional targets of estrogen signaling were determined by RNAseq. Finally, the receptor status for ER $\alpha, E R \beta$ and PR was determined in a panel of HGSC cell lines. Our results highlight the need to consider the $\mathrm{E}_{2}$ response of putative progenitor cell populations of HGSC to investigate estrogen's role in initiation and progression of OVCA. The occurrence and survival rates for OVCA have not improved in over 40 years emphasizing the necessity for better understanding and effectively treating or preventing this deadly disease. This study demonstrates that estrogen receptor activates unique oviduct-specific targets that may provide new targets for preventing or treating fallopian tube derived tumors.

\section{Methods}

\section{Cell culture}

MOE and MOSE cells (passages 7-25) were cultured as previously described $[16,17]$. For experiments investigating $E_{2}$ and SERM response, cells were cultured at least $48 \mathrm{~h}$ in "stripped media" consisting of phenol red free $\alpha$-modified Eagle's medium (Life Technologies, Carlsbad, CA) supplemented with $10 \% \mathrm{v} / \mathrm{v}$ charcoal stripped fetal bovine serum (FBS) (Life Technologies) [18], $1 \mathrm{mg} / \mathrm{mL}$ gentamycin (Mediatech, Manassas, VA), $2 \mathrm{mM}$ L-glutamine (Life Technologies), $100 \mathrm{U} / \mathrm{mL}$ penicillin and $50 \mu \mathrm{g} / \mathrm{mL}$ streptomycin (Roche, Indianapolis, IN) prior to treatments. KURAMOCHI (passages 15-25), OVSAHO (passages 45-60) and OVKATE (passages 45-60) cell lines were purchased from the JCRB Cell bank and maintained in RPMI1640 (Mediatech) supplemented with $10 \% \mathrm{FBS}, 100 \mathrm{U} / \mathrm{mL}$ penicillin and $50 \mu \mathrm{g} / \mathrm{mL}$ streptomycin. OVSAHO were cultured for 48 hours (h) prior to treatment and treated in phenol-red free RPMI1640 (Life Technologies) supplemented with $10 \%$ charcoal stripped FBS, $100 \mathrm{U} / \mathrm{mL}$ penicillin and $50 \mu \mathrm{g} / \mathrm{mL}$ streptomycin. OVCAR4 (passages 15-30) were acquired from the National Cancer Institute Division of Cancer Treatment and Diagnosis Tumor Repository and maintained in the same media as KURAMOCHI plus $1 \%$ l-glutamine. SKOV3 (passages 10-30) and OVCAR3 (passages 3-30) cell lines were acquired from ATCC (Manassas, VA). SKOV3 were cultured in McCoy's 5A supplemented with $2.3 \mathrm{~g} / \mathrm{L}$ sodium carbonate, $10 \%$ FBS, $100 \mathrm{U} / \mathrm{mL}$ penicillin and $50 \mu \mathrm{g} / \mathrm{mL}$ streptomycin. OVCAR3 were maintained in minimum essential media supplemented with $20 \%$ FBS, $1 \%$ tglutamine, $1 \%$ non-essential amino acids, $1 \%$ sodium pyruvate, $100 \mathrm{U} / \mathrm{mL}$ penicillin and $50 \mu \mathrm{g} / \mathrm{mL}$ streptomycin. Cell lines were authenticated by STR analysis at DDC Medical (murine) or UIC DNA Services Facility (human).

\section{Western blotting}

Cells were plated at a density of $10-30 \times 10^{4}$ cells per well in a 6-well dish in stripped media. Twenty-four hours post plating cells were washed with PBS and the media was replenished. After another $24 \mathrm{~h}$, cells were washed with PBS and treated with DMSO $(0.1 \%)$ or compounds for indicated times.

Following treatment, cells were washed with PBS then harvested on ice in RIPA buffer $(50 \mathrm{mM}$ Tris, $\mathrm{pH} 7.6$, $150 \mathrm{mM} \mathrm{NaCl}, 1 \% \mathrm{v} / \mathrm{v}$ Triton X-100, $0.1 \% \mathrm{w} / \mathrm{v}$ sodium dodecyl sulfate) and frozen at $-80{ }^{\circ} \mathrm{C}$. Lysates were 
centrifuged at 14,000 rpm for $5 \mathrm{~min}$ and clarified samples were quantified by BCA (Pierce, Rockford, IL), separated by SDS PAGE $(8 \%)$ at $100 \mathrm{~V}$ for $2.5 \mathrm{~h}$ then transferred for $2 \mathrm{~h}$ at $25 \mathrm{~V}$ to nitrocellulose (GE Healthcare Bio-Sciences, Pittsburgh, PA). Blots were blocked in $5 \%$ milk TBS-T (Tris Buffered Saline-Tween 20) for $1 \mathrm{~h}$ at room temperature (RT) followed by overnight incubation at $4{ }^{\circ} \mathrm{C}$ in primary antibodies: Anti-ER $\alpha$ (1:300, MC20 or 1:200 HC-20, Santa Cruz, Dallas, TX), Anti-PR (1:500 H-190, Santa Cruz), Anti-actin (1:1000, SigmaAldrich). Following 3 washes in TBS-T, blots were incubated in Anti-rabbit horseradish peroxidase-conjugated secondary (1:1000, Cell Signaling, Cambridge, MA) for 30 min at RT. After secondary, blots were washed $3 \mathrm{X}$ in TBS-T then bands were imaged using SuperSignal West Femto Chemiluminescent Substrate (Pierce) on a FluorChem E Imager (ProteinSimple, Santa Clara, CA). Densitometry was performed using Protein Simple software.

\section{qPCR}

Cells were treated as described for Western blots, then harvested in $500 \mu \mathrm{L}$ of Trizol Reagent (Life Technologies) and frozen at -80 until processed according to manufacturer's protocol. Purified RNA was treated with DNAseI (New England Biolabs, Ipswich, MA) for $10 \mathrm{~min}$, followed by heat inactivation. $1 \mu \mathrm{g}$ of purified RNA was reverse transcribed using Revertaid Reverse Transcriptase (Thermo Fisher Scientific, Waltham, MA) in the presence of Ribolock RNAse Inhibitor (Thermo Fisher Scientific). Expression was monitored using Faststart Universal Sybr Green (Rox) (Roche) using protocol: $10 \mathrm{~min}$ at $94{ }^{\circ} \mathrm{C}, 40$ cycles of $10 \mathrm{sec}$ at $94{ }^{\circ} \mathrm{C}, 30 \mathrm{sec}$ at $60{ }^{\circ} \mathrm{C}$ followed by a melt curve. Primers listed in Additional file 1: Table S1.

\section{Immunofluorescence}

MOE cells were cultured in charcoal stripped media for $72 \mathrm{~h}$ and then plated (50,000 per well) onto Millicell EZ slides (Millipore, Billerica, MA). The next day the cells were fixed with $4 \%$ paraformaldehyde and probed with primary antibody against estrogen receptor $\alpha$ (1:200, MC-20, Santa Cruz) with $10 \%$ goat serum. Slides washed and probed with secondary antibody (AlexaFluro 594 A11037, Life Technologies) before mounting with DAPI containing mounting media $(\mathrm{H}-1500$, Vector Laboratories, Burlingame, CA). Images were captured with a Nikon Eclipse E600 microscope at 40x.

\section{Proliferation assay}

Cells were cultured in stripped media for $72 \mathrm{~h}$ followed by passaging and plating of 1,000 cells/well in a 96 well plate. Twenty-four hours post plating, cells were treated with solvent or compounds for $72 \mathrm{~h}$ then fixed with
$20 \% \mathrm{w} / \mathrm{v}$ Trichloroacetic acid for $24 \mathrm{~h}$ at $4{ }^{\circ} \mathrm{C}$ and processed for sulforhodamine $\mathrm{B}$ colorimetric assay as described [19]. Absorbance at $505 \mathrm{~nm}$ was measured using a BioTek Synergy MX microplate reader (BioTek, Winooski, VT).

\section{Wound healing assay}

Cells were cultured for $72 \mathrm{~h}$ as described in the proliferation assay, followed by passaging and plating of $6 \times 10^{4}$ per well in a 24 well plate. Twenty-four hours post plating a pipette tip induced a scratch across the monolayer of cells. Images of the wound were taken at time zero and $24 \mathrm{~h}$ post scratch. The percent closure of the wound was calculated as the area of the scratch at $24 \mathrm{~h}$ post scratch divided by the area of the scratch at time zero using ImageJ (National Institutes of Health).

\section{Luciferase assay}

SKOV3 cells were trypsinized and plated in 24-well plate $\left(3.5 \times 10^{4}\right.$ cells/ well $)$ in stripped media. Incubation of cells with pERE-luciferase plasmid (100 ng/well) [20], RSV- $\beta$-galactosidase (100 ng/well, [21], and TransIT LT1 transfection reagent $(1 \mu \mathrm{L}$ per well, Mirus Bio, Madison, WI) was performed overnight in fresh media then treated for $24 \mathrm{~h}$. Luciferase production and $\beta$-galactosidase activity (for transfection normalization) were measured as described previously [21].

\section{RNAseq library construction and sequencing}

Cells were treated as described in qPCR assay, followed by RNA isolation using Qiagen Qiashredder column, on column DNAse treatment and Qiagen RNAeasy spin columns (Qiagen, Valencia, CA). Library construction and sequencing were performed at the Genomics Core facility at the University of Chicago. RNA quality and quantity were determined with the Agilent Bioanalyzer 2100 , with RNA integrity numbers (RIN) of 10 and quantities of $100 \mathrm{ng}$ or more per sample. Samples were enriched for mRNA using oligo-dT columns. Directional $50 \mathrm{bp}$ single-end mRNA libraries were prepared using Illumina TruSeq mRNA Sample Preparation Kits per manufacturer's instructions. Briefly, polyadenylated mRNAs were captured from total RNA using oligo-dT selection. Next, samples were converted to cDNA by reverse transcription, and each sample was ligated to Illumina sequencing adapters containing unique barcode sequences. Barcoded samples were then amplified by PCR and the resulting CDNA libraries quantified using qPCR. Finally, equimolar concentrations of each cDNA library were pooled and sequenced on the Illumina HiSeq2500. 


\section{Transcriptome analysis}

The quality of DNA reads, in fastq format, was evaluated using FastQC. Adapters were removed and reads of poor quality filtered. The data was processed largely following the procedure described in [22]. Briefly, reads were aligned to the Mus musculus genome (mm10) using TopHat (v2.0.8b). Subsequently, aligned reads, in conjunction with a gene annotation file for $\mathrm{mm} 10$ obtained from the UCSC website, were used to determine the expression of known genes using Cufflinks (v2.1.1). Individual transcript files generated by Cufflinks for each sample were merged into a single gene annotation file, which was then used to perform a differential expression analysis with the Cufflinks routine, cuffdiff. Differential expression was determined by cuffdiff using the procedure described in Trapnell et al [22], using an FDR cutoff value of 0.05 . Results of the differential expression analysis were processed with cummeRbund. Differentially expressed genes were separated into upregulated and downregulated lists. A pathway analysis was performed on both gene lists using GeneCoDis [23-25] to identify pathways enriched with genes that were upregulated and downregulated.

\section{Statistical analysis}

Data shown are represented as the mean of at least three experiments, with errors bars representing the standard error. Statistical analysis was conducted with GraphPad Prism (GraphPad, La Jolla, CA) using one-way ANOVA with a Tukey's post hoc test.

\section{Results}

\section{Putative OVCA progenitor cell type estrogen responsive}

The fallopian tube (oviduct in the mouse) epithelium is likely one of the sources of HGSC. To investigate the role of estrogen signaling in this precursor cell type of HGSC, we evaluated the response of murine oviductal epithelium (MOE) cells derived from CD1 and FVB murine backgrounds subjected to 17-beta-estradiol $\left(\mathrm{E}_{2}\right)$ treatment (Fig. 1a, b). CD1 MOE cells are a polyclonal cell line consisting of both secretory and ciliated oviductal epithelial cells [16]. The FVB MOE cells are monoclonal, comprised exclusively of secretory oviductal epithelial cells [17]. The disappearance of ER $\alpha$ via proteasome-mediated proteolysis [26], and upregulation of the canonical ER regulated target progesterone receptor (PRA and PRB, two isoforms encoded by the Pgr gene) were monitored for $E_{2}$ responsiveness via Western blot analysis. Immunofluorescence revealed that $100 \%$ of FVB MOE cells expressed ER $\alpha$ (Fig. 1e). MOE cell lines demonstrated robust $E_{2}$ responsiveness for these endpoints.

HGSC is a heterogeneous disease, the only common alteration ( $<96 \%$ of cases) being a mutation in the $T p 53$ gene [27]. Intriguingly, FVB MOE cells stably transfected with a plasmid encoding the human Tp53 gene mutated at $\mathrm{R} 273 \mathrm{H}$ [17] expressed elevated protein levels of both ER $\alpha$ and PRA/PRB (Fig. 1b), although the transcriptional strength of $\mathrm{PR}$ induction by $\mathrm{E}_{2}$ was not significantly different than observed in wildtype MOE FVB cells (Additional file 2: Figure S1a-c).

A human fallopian tube secretory epithelial cell (FTSEC) line [28] did not express detectable ER $\alpha$ and $\mathrm{PR}$, precluding study of $\mathrm{E}_{2}$ responsiveness in human cells (Fig. 1c), although transient transfection of a plasmid encoding $E R \alpha$ did recover the ability for $E_{2}$ to induce transcription of Pgr (data not shown). Continuous culturing of the CD1 MOE cell line resulted in a decrease of the receptors (Fig. 1d) suggesting growth on plastic is capable of inducing receptor loss. These results were similar to a baboon FTSEC that also lost receptor in culture that could be reactivated [20].

The $E_{2}$ responsiveness of the classically studied OVCA precursor, the ovarian surface epithelium (OSE) was also investigated. A murine ovarian surface epithelium (MOSE) cell line from the FVB background [16], expressed much less ER $\alpha$ and PRA/PRB compared to the MOE cell lines, and further PRA/PRB levels were not altered by $E_{2}$ treatment (Fig. 1b). OSE are known to express the receptors in vivo [29], once again indicating culturing on tissue culture plastic could lead to receptor depletion. Attempts to enhance receptor expression and $\mathrm{E}_{2}$ responsiveness of MOSE cells using an HDAC inhibitor (HD13) [30] or the demethyltransferase inhibitor, 5-azacytidine were unsuccessful (Additional file 2: Figure S1d and data not shown).

\section{SERMs antagonize $E_{2}$ in MOE}

Women at high risk for breast cancer and OVCA, having a mutation in either BRCA1 or BRCA2 genes, may prophylactically take selective estrogen receptor modulators (SERMs), such as 4-hydroxytamoxifen (4OHT), to decrease their risk of developing breast cancer [31]. SERM action as an ER agonist or antagonist is cell type specific and the effect on ER and the response of the fallopian tube epithelium in terms of cancer biology to SERMs is not well documented. MOE cells were used to elucidate whether the SERMs 4OHT, raloxifene (RAL) and desmethylarzoxifene (DMA), function as ER antagonists or agonists. QPCR analysis of the genes $P g r$ and Greb1, revealed significant $(p<0.001)$ induction in response to $E_{2}$ in both CD1 and FVB MOE cells (Fig. 2a, b and Additional file 3: Figure S2a,b). All SERMs tested did not significantly activate Pgr or Greb1 compared to the DMSO but did significantly $(p<0.001)$ antagonize $\mathrm{E}_{2}$ dependent induction in both MOE cell lines (Fig. 2a, b and Additional file 3: Figure S2a,b). Western blot analysis of CD1 MOE treated with $E_{2}$ and SERMs revealed 

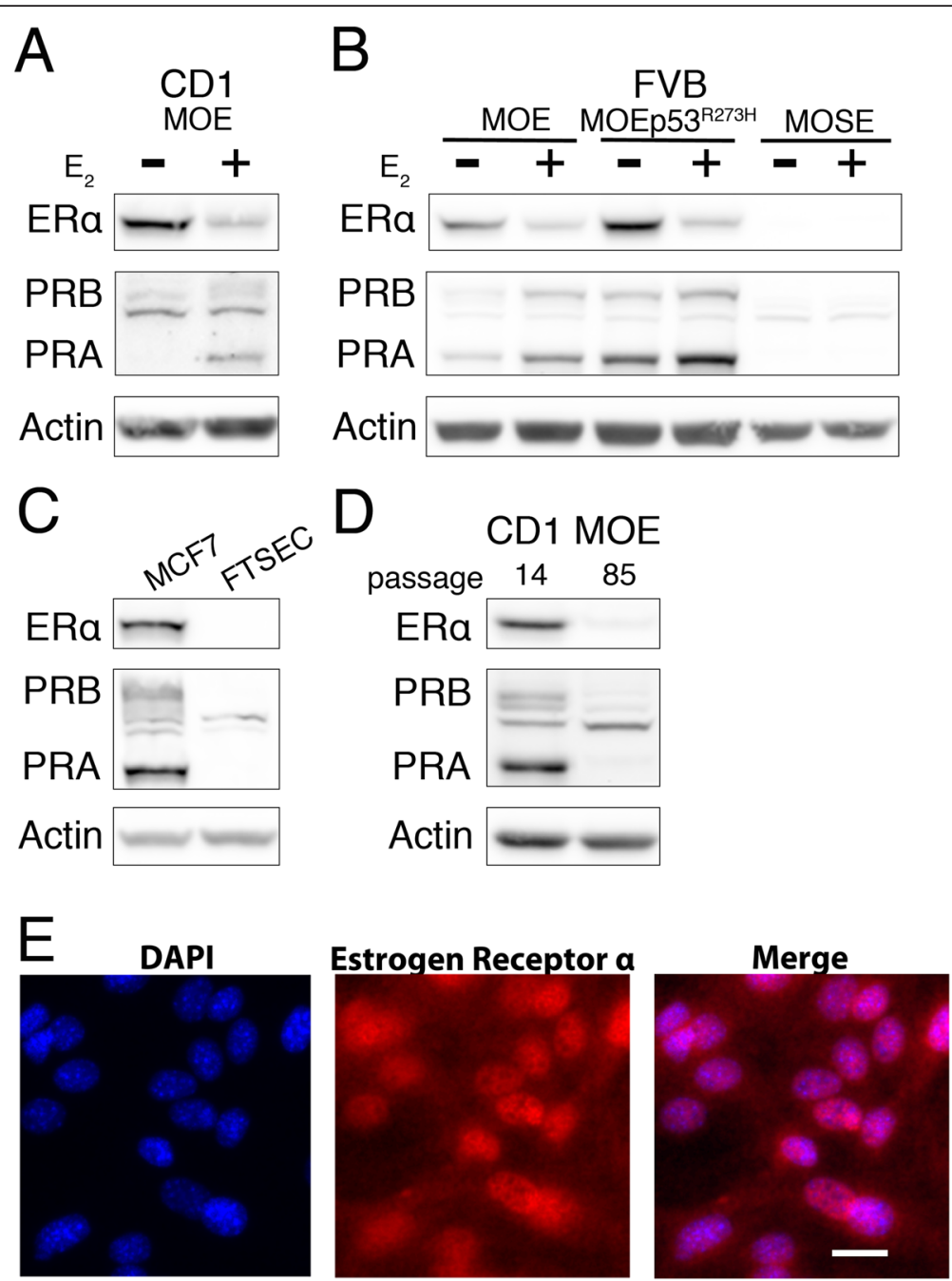

Fig. 1 Receptor status and estrogen responsiveness monitored by Western blot analysis. a Analysis of ERa and PR expression in response to $24 \mathrm{~h}$ $17 \beta$-estradiol $\left(1 \mathrm{nM}, \mathrm{E}_{2}\right)$ treatment in CD1 MOE cells or (b) FVB MOE and MOSE cells. c Western blot analysis of human fallopian tube secretory epithelial cells (FTSEC) and receptor positive MCF7 breast cancer cells. $\mathbf{d}$ Receptor protein levels of early passage (P14) and late passage (P85) Cd1 MOE cells. e Immunofluorescence in FVB MOE cells for ERa and DAPI counterstain. Scale bar $=20 \mu \mathrm{m}$

significant $(P<0.001)$ PR induction and disappearance of $E R \alpha$ in response to $E_{2}$ treatment (Fig. 2c, d, e). These findings indicate that the SERMs antagonize $\mathrm{E}_{2}$ in oviduct epithelial cells.

$E_{2}$ increases the growth rate in a subset of ER positive OVCA cell lines $[32,33]$. The reported functional response of fallopian tube epithelial cells to estrogen signaling involves triggering the differentiation of epithelial cells, protein secretion, and regulation of cilia beating [34]. In vivo proliferation rates have not been reported to change due to ovulation, which has high levels of estrogen in the follicular phase of the cycle. To evaluate the functional response of MOE cells to $E_{2}$ and SERMs, a proliferation assay was employed. Following 72-hour treatment, a small but significant $(p<0.05)$ proliferation increase was observed in the presence of $E_{2}$ but not
SERMs in CD1 MOE cells (Fig. 3a). This $E_{2}$ dependent proliferative increase was blocked by all the SERMs tested. No $E_{2}$ dependent growth increase was observed in the FVB MOEs suggesting a strain specific $E_{2}$ responsiveness (Additional file 3: Figure S2b).

To determine if $E_{2}$ and SERMs alters the migration of MOE cells, a wound-healing assay was utilized. No difference in migration was observed for either MOE cell line (Fig. 3b and Additional file 3: Figure S2c), suggesting $E_{2}$ does not enhance the migration of normal oviductal epithelial cells.

\section{RNAseq of MOE cells}

The particular set of genes regulated by $E_{2}$ is cell type specific [15]. A number of $E_{2}$-regulated genes in breast cancer cell lines were not altered by $E_{2}$ 

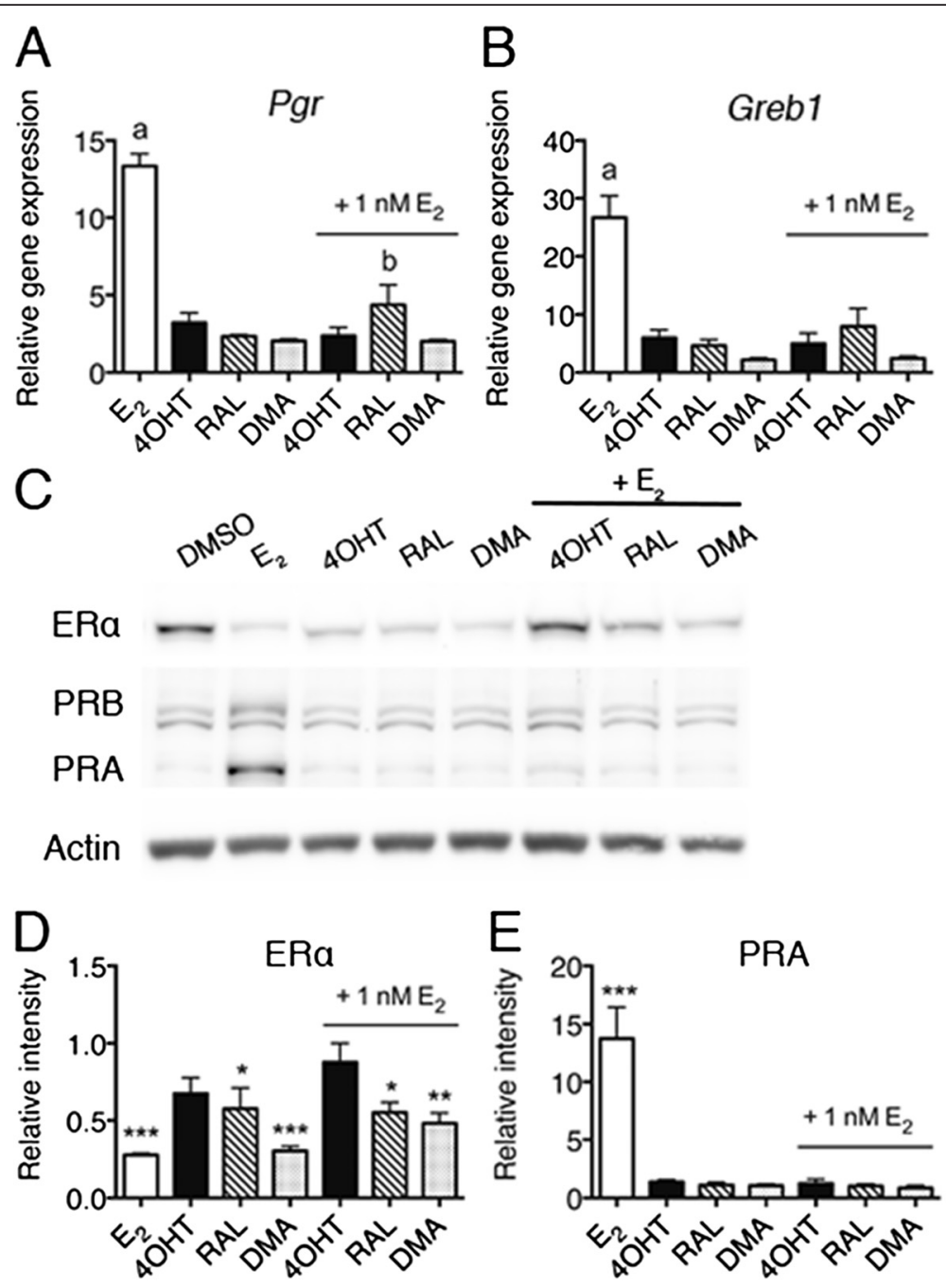

Fig. 2 SERMs antagonize $E_{2}$ in CD1 MOE. a, b qPCR analysis of (a) Pgr and (b) Greb1 induction in response to $48 \mathrm{~h}$ hormone starvation and $24 \mathrm{~h}$ treatment with DMSO, $1 \mathrm{nM} E_{2}$ or 100 nM SERMs. "a" indicates significant difference compared to all treatments $(p<0.001)$ and "b" is significantly different than DMSO control treatment $(p<0.01)$. c Western blot analysis of CD1 MOE treated for $24 \mathrm{~h}$ with $1 \mathrm{nM} \mathrm{E}_{2}$ and $100 \mathrm{nM}$ SERMs and the combination. d, e Densitometry of ERa and PRA bands relative to solvent treated cells normalized to actin. ERa is significantly lower in $E_{2}$, RAL and DMA treated cells, while $4 \mathrm{OHT}$ does not result in ERa degradation. PR is significantly upregulated in $E_{2}$ treated cells compared to all conditions. Significance determined by one-way ANOVA followed by Tukey's post hoc test where * indicates $p<0.05,{ }^{* *}$ indicates $p<0.01,{ }^{* * *}$ indicates $p<0.001$

treatment in MOE cells, for example Nrip1 (Additional file 3: Figure S2e) [35]. To identify $\mathrm{E}_{2}$ regulated genes in the oviduct epithelia, RNAseq was performed on FVB MOE cells treated for $24 \mathrm{~h}$ with solvent (DMSO), $1 \mathrm{nM} \mathrm{E}_{2}$ or $100 \mathrm{nM} 4 \mathrm{OHT}$ in triplicate. The FVB MOE cell line was utilized for two reasons. First, HGSC is thought to arise from the secretory cells of the fallopian tube. The FVB MOE line is a monoclonal cell line of exclusively secretory cells [17]. Second, the cell model was chosen instead of primary oviduct culture since primary cells would have contaminating ciliated and underlying stromal cells that could confound the results [36]. Over 438 million reads between the 9 samples (between 40-60 million per sample) were sequenced using Illumina HiSeq2500 platform (Additional file 1: Table S2).

Analysis of the dataset identified 314 gene targets differentially expressed (287 upregulated and 28 downregulated, FDR adjusted $p$-value $<0.05)$ in response to $\mathrm{E}_{2}$ compared to DMSO treated MOE cells. The top 15 genes significantly up and down regulated are listed in Table 1 and 2, respectively. Few genes were shown to be differentially expressed between DMSO and 4OHT (Additional file 1: Table S3 and Additional file 1: Table S4) and only 4 were $4 \mathrm{OHT}$ specific, further suggesting that $4 \mathrm{OHT}$ functions as an ER antagonist in this cell type. 274 genes targets were differentially expressed between $\mathrm{E}_{2}$ and $4 \mathrm{OHT}$ treated MOE cells. Figure 4a 


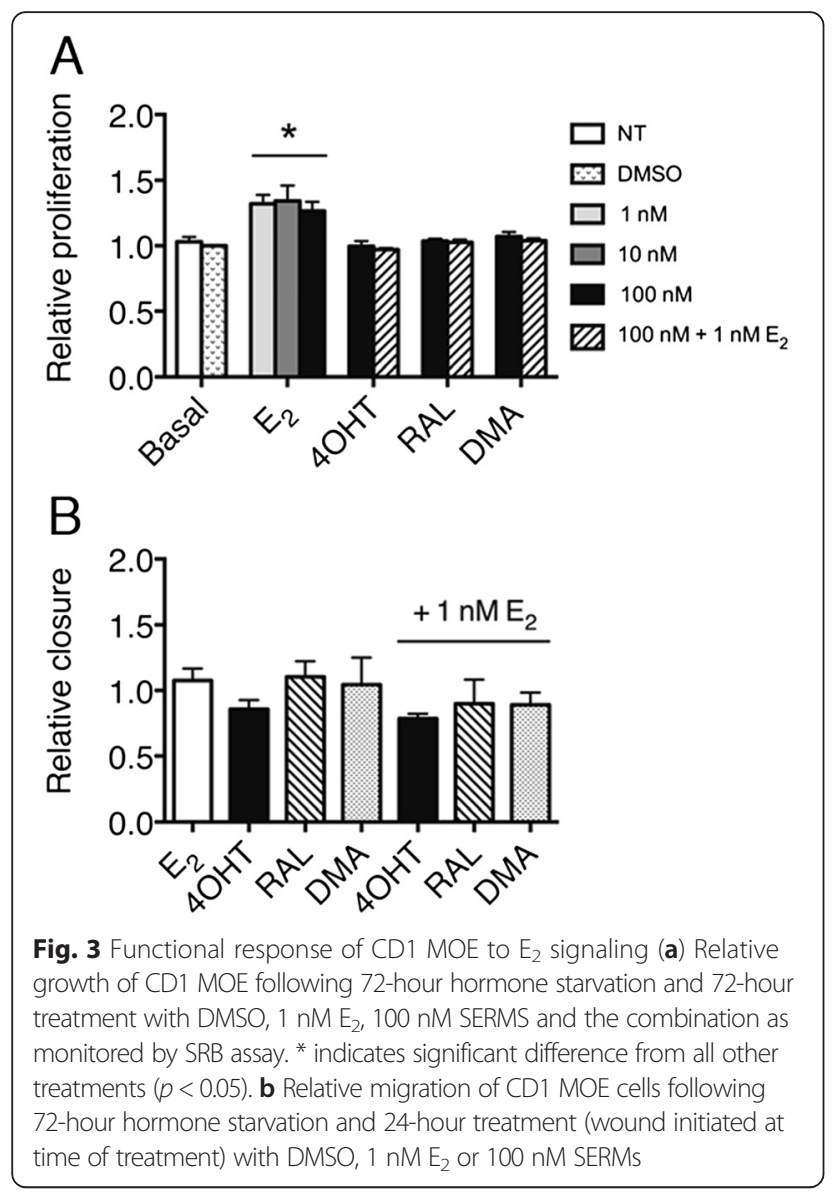

illustrates the number of regulated genes in common between each condition, highlighting a large overlap in genes differentially regulated by $\mathrm{E}_{2}$ compared to DMSO and 4OHT. A subset of genes, Csf2, Dhrs9, and Dcn were validated for response to $\mathrm{E}_{2}$ and $4 \mathrm{OHT}$ by qPCR (Fig. 4b-d).

GO analysis of the upregulated genes in response to $\mathrm{E}_{2}$ identified a number of biological processes. The heat map shown in Fig. 4e reflect changes in expression between DMSO versus $\mathrm{E}_{2}$ or $4 \mathrm{OHT}$ for the two most enriched biological processes. Regulation of proliferation was the largest group identified. Regulation of apoptosis was the second highest group enriched. Other significantly enriched processes include response to steroid hormone stimulus, mammary gland epithelium development and calcium ion homeostasis.

\section{OVCA cell line receptor status}

Estrogen only hormone replacement therapy and the number of lifetime ovulations are associated with OVCA [8] . The SKOV3 cell line is the most cited OVCA cell line [37]. Previous reports indicate that SKOV3 are not growth responsive to $E_{2}$ due to expression of a truncated ER $\alpha$ [38]. While SKOV3 were responsive to $\mathrm{E}_{2}$ by a reporter assay containing an EREbinding element fused to a luciferase gene (Fig. 5a), they were not at the endogenously regulated $P G R$ gene by either qPCR or Western blot analysis (Figs. 5b, c). Another commonly used $E_{2}$-responsive cell line in the OVCA literature, BG1, was reported to proliferate in response to estrogen, although a subset of studies using this cell line has subsequently been identified as MCF7 breast cancer cells [39]. Currently, neither cell type are

Table 1 Top 15 up-regulated genes between DMSO and $E_{2}$ treated MOE cells

\begin{tabular}{llcc}
\hline Gene & Description & Log2 fold change & FDR adjusted $p$-value \\
\hline Bpifc & BPI fold containing family C & 4.01 & $5.31 \mathrm{E}-03$ \\
Gbp8 & Guanylate-binding protein 8 & 3.36 & $5.31 \mathrm{E}-03$ \\
Csf2 & Colony stimulating factor 2 (granulocyte-macrophage) & 3.30 & $5.31 \mathrm{E}-03$ \\
Cldn10 & Claudin 10 & 3.21 & $5.31 \mathrm{E}-03$ \\
Ptprz1 & Protein tyrosine phosphatase, receptor type Z, polypeptide 1 & 3.13 & $5.31 \mathrm{E}-03$ \\
Dhrs9 & Dehydrogenase/reductase (SDR family) member 9 & 3.10 & $5.31 \mathrm{E}-03$ \\
Oit1 & Oncoprotein induced transcript 1 & 2.65 & $5.31 \mathrm{E}-03$ \\
Wasf3 & WAS protein family, member 3 & 2.65 & $5.31 \mathrm{E}-03$ \\
Bcas1 & Breast carcinoma amplified sequence 1 & 2.62 & $5.31 \mathrm{E}-03$ \\
Pgr & Progesterone receptor & 2.61 & $5.31 \mathrm{E}-03$ \\
Dcn & Decorin & 2.51 & $5.31 \mathrm{E}-03$ \\
F5 & Coagulation factor V & 2.39 & $5.31 \mathrm{E}-03$ \\
Krtap1-5 & Keratin associated protein 1-5 & 2.33 & $5.31 \mathrm{E}-03$ \\
Padi2 & Peptidyl arginine deiminase, type II & 2.32 & $5.31 \mathrm{E}-03$ \\
Padi1 & Peptidyl arginine deiminase, type I & 2.31 & $5.31 \mathrm{E}-03$ \\
\hline
\end{tabular}


Table 2 Top 15 down-regulated genes between DMSO and $E_{2}$ treated MOE cells

\begin{tabular}{llcc}
\hline Gene & Description & Log2 fold change & FDR adjusted $p$-value \\
\hline H19 & H19, imprinted maternally expressed transcript & -1.65 & 0.02 \\
Mep1a & Meprin 1 alpha & -1.48 & 0.03 \\
Rgs8 & Regulator of G-protein signaling 8 & -1.35 & 0.05 \\
Hhip & Hedgehog-interacting protein & -1.26 & 0.01 \\
G6pc2 & Glucose-6-phosphatase, catalytic, 2 & -1.14 & 0.01 \\
Adamts16 & A disintegrin-like and metallopeptidase (reprolysin type) with & -1.09 & 0.01 \\
& thrombospondin type 1 motif, 16 & -1.05 & 0.01 \\
Apln & Apelin & -0.88 & 0.02 \\
Cyp26a1 & Cytochrome P450, family 26, subfamily a, polypeptide 1 & 0.05 \\
Upk3b & Uroplakin 3B & -0.83 & 0.03 \\
Aqp1 & Aquaporin 1 & -0.70 & 0.05 \\
Nup210 & Nucleoporin 210 & -0.65 & 0.02 \\
Tgfbr3 & Transforming growth factor, beta receptor III & -0.64 & 0.01 \\
I830012O16 Rik, Ifit3 & RlKEN cDNA 1830012O16 gene & -0.63 & 0.01 \\
Havcr1 & Hepatitis A virus cellular receptor 1 & -0.62 & 0.01 \\
Pdk4 & Pyruvate dehydrogenase kinase, isoenzyme 4 & -0.62 & \\
\hline
\end{tabular}

genomically validated models of HGSC, thought to develop from fallopian tube cells [37] and highlight the need to explore additional cell models.

We analyzed a number of representative and commercially available HGSC lines for the presence of the steroid hormone receptors ER $\alpha$, PR, and ER $\beta$. Molecular profiling of these cell lines predict OVSAHO, Kuramochi, OVCAR4 and OVKATE are likely, while OVCAR3 is possibly and SKOV3 is unlikely to be HGSC [37]. All OVCA cell lines tested expressed much less or no ER $\alpha$ and PR compared to the receptor positive breast cancer cell line MCF7 by both Western blot and RNA analyses (Fig. $5 \mathrm{~d}$-e). ER $\beta$ status was monitored for all samples by qPCR revealing minimally detectable amounts mRNA (Fig. 5e, a cell line expressing a plasmid encoding ER $\beta$ was used to validate the primers). The dearth of ER and PR receptors in OVCA cell lines is in contrast to the $60 \%$ of ER positive tumors [13] and makes investigation into the role of estrogen in OVCA challenging.

Despite low levels of ER $\alpha$ and PR in the OVCA cell lines, one HGSC cell line, OVSAHO, which expressed $E R \alpha$ and $P R$ was tested for $E_{2}$ responsiveness. OVSAHO proliferation was significantly increased by $\mathrm{E}_{2}$, but not SERMs, following a 7 day treatment, and the SERMs antagonized $E_{2}$ dependent growth (Fig. 5f). Following 24-hour treatment, a mild albeit not significant induction was observed at the $P G R$ gene in response to $E_{2}, 4 \mathrm{OHT}$ and $\mathrm{E}_{2}$ plus $4 \mathrm{OHT}$ (Fig. 5g). Therefore, despite the lower levels of receptors compared to MCF7 breast cancer cells, OVSAHO are an estrogen responsive cell model.

\section{Discussion}

The epithelium of the fallopian tube is considered to be one of the origins of HGSC and estrogen replacement therapy impacts the risk of OVCA. This study characterized the agonist/antagonist function of SERMs in a normal murine model of fallopian tube epithelium, identified the genes regulated by estrogen signaling in this cell type to shed light on the subset of genes regulated by $E_{2}$ in this cell type, and determined the $E_{2}$ responsiveness of a panel of HGSC cell lines.

\section{SERMS antagonize $E_{2}$ in MOE cells}

Tamoxifen is a common treatment for receptor positive breast cancer, as well as given prophylactically for women at high risk for developing breast cancer [31]. Given the risk associated with Tamoxifen for the uterus, the response of Tamoxifen and other SERMs in the fallopian tube epithelium is highly important for clarifying the risks of using these drugs in women with intact fallopian tubes. Responses such as proliferation or migration has not been well documented in fallopian tube epithelium as compared to the response in the breast or uterine tissue, traditional hormone responsive tumor types [20]. Reports from our lab indicate that estrogen did not proliferate oviductal epithelium in FVB strains, or in organotypic cultures of the mouse, baboon, or human $[20,40]$. Long term (at least 4 years) Tamoxifen treatment of women with breast cancer has been reported to increase tubal dysplasia [41]. The anti-estrogen effect reported here on isolated epithelial cell lines suggests the dysplastic effect of tamoxifen may not be due to 


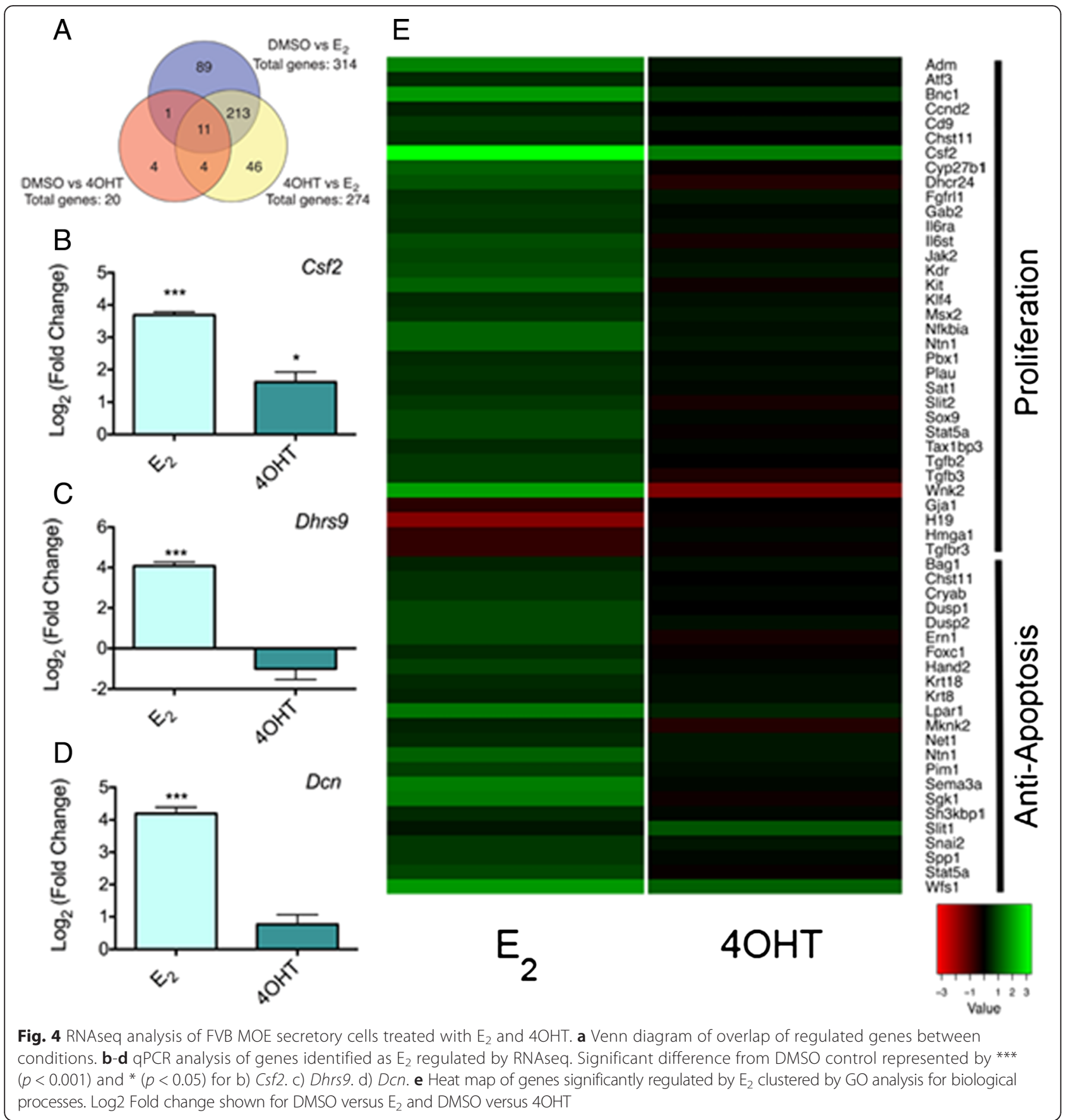

proliferation, but rather the length of exposure, or a non transcriptional effects, such as DNA damage [42].

\section{RNAseq identifies targets of $E_{2}$ signaling in MOE cells}

Many $E_{2}$ regulated genes in other normal and cancer cell types were also regulated in MOE cells including $P g r$, Greb1, Csf2, and Dhrs9 [43, 44], while other $\mathrm{E}_{2}$ regulated genes in MCF7 cells were not regulated (Nrip1) [35] or even expressed (Tff1) in MOE cells [45]. Interestingly, in contrast to MCF7 [45], the majority of differentially expressed genes were upregulated in MOE cells at the time point $(24 \mathrm{~h})$ probed. Table 1 and 2 lists the most highly regulated genes in response to $E_{2}$ in MOE cells, some of which are relatively uncharacterized. BPI fold containing family $\mathrm{C}$ gene $(B p i f c)$, involved in lipid binding [46], demonstrated the largest induction in response to $E_{2}$. One gene specifically upregulated by $4 \mathrm{OHT}$ was the RNA component of mitochondrial processing endonuclease (Rmrp), reported to be upregulated in $4 \mathrm{OHT}$ resistant as compared to $4 \mathrm{OHT}$ sensitive breast cancer 


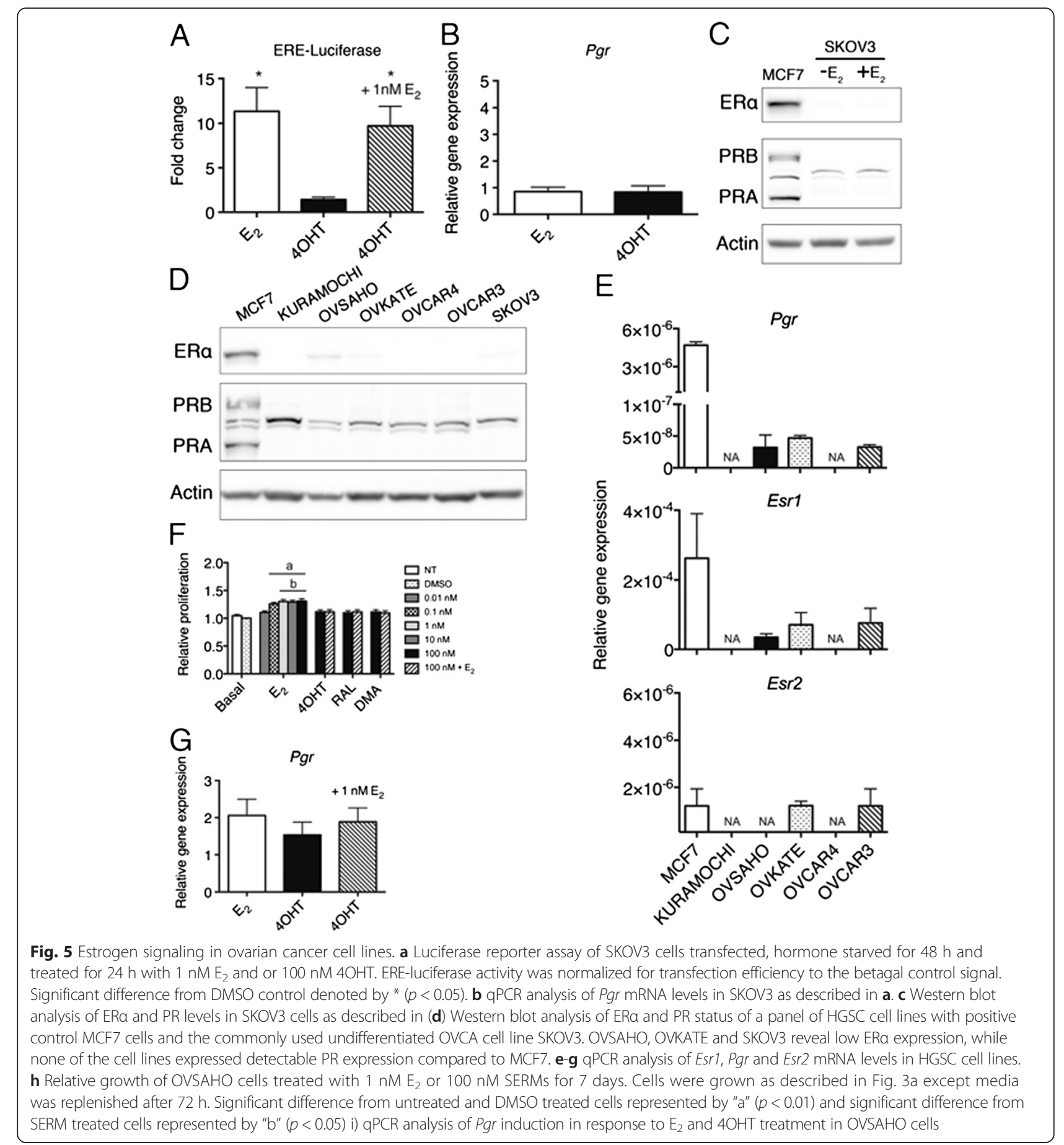

cells [47]. The biological processes enriched in MOE cells included regulation of proliferation and apoptosis, response to hormone stimulus and calcium ion homeostasis. The RNAseq analysis identified genes responsible for both positive and negative regulation of proliferation, which may reconcile the lack of significant proliferative increase in the FVB MOE background. In the fallopian tube, calcium is required for sperm capacitation [48] and cilia beating [49], therefore $E_{2}$ may regulate calcium levels in the fallopian tube as part of reproductive biology.

Comparison of the MOE $\mathrm{E}_{2}$ responsive genes and The Cancer Genome Atlas of ovarian cancer tumors identified a number of genes with significance in OVCA in common between the two groups [27, 50, 51]. For example, $E_{2}$ increased expression of the cyclin dependent kinase 2 (Ccnd2) gene, which is altered (via amplification or mRNA upregulation) in $12 \%$ of ovarian tumors, and 
has been shown to be upregulated in some OVCAs [52]. $J a k 2$, and Kit were also upregulated in response to $\mathrm{E}_{2}$ and altered in $12 \%$ and $8 \%$ of ovarian tumors, respectively. The St3gal1 gene, encoding a glycosyltransferase, was upregulated by $E_{2}$ and altered (mostly amplified or showing mRNA upregulation) in $30 \%$ of ovarian tumors. The significance of ST3Gal1 in OVCA is unknown, but has been linked to colon cancer [53]. Cyclin dependent kinase 1 (Ccnd1) was downregulated in response to $E_{2}$ and altered in $8 \%$ of ovarian tumors. Interestingly, CCND1 is overexpressed in cisplatin resistant testicular cancer and OVCA [54]. The overlap of genes regulated by $E_{2}$ in MOE cells and alteration in OVCA provides a number of potential new targets for further investigation of $\mathrm{E}_{2}$ regulation in OVCA.

\section{Need for better $E_{2}$ responsive models of HGSC}

The most frequently used estrogen responsive OVCA cell lines are not ideal models of HGSC including SKOV3 and the NIEHs BG1 cells [38, 39]. Two other estrogen responsive OVCA cell lines, PEO1 and PEO4, have recently been reported as HGSC [55]. These cell lines proliferate in response to $E_{2}$ in culture and xenografts and $\mathrm{E}_{2}$ increases risk of distant metastases [32, 33]. The HGSC cell lines investigated in this study express much less ER and PR receptors compared to the estrogen responsive MCF7. Nevertheless, the likely HGSC cell line OVSAHO proliferates in response to $E_{2}$, but not SERMs. Further validation of other/more estrogen responsive HGSC cell lines is desperately needed to aid in understanding the role of estrogen in OVCA and whether ER expressing HGSC would respond to anti-estrogen therapy. By studying how the cancers and different progenitors, such as the oviductal or OSE, respond to estrogen may aid in the use of SERMs in tumors that express ER or help to uncover if long-term use of Tamoxifen could enhance dysplastic lesions in the fallopian tube.

\section{Conclusion}

This study shows that the fallopian tube epithelia respond to $E_{2}$ stimulation by regulating expression of a tissue-specific set of target genes. All SERMs tested inhibited $E_{2}$-stimulated responses, showing SERMs are antagonistic action in the fallopian tube. Thus direct effects of $E_{2}$ on the fallopian tube epithelium may play a role in the development of ovarian cancer.

\section{Ethics approval}

Not Applicable.

\section{Consent for publication}

Not applicable.

\section{Availability of data and material}

The sequences and expression values reported in this paper have been deposited in the NCBI GEO database, Accession number GSE67207.

\section{Additional files}

\begin{abstract}
Additional file 1: Table S1. Primers used in study. Table S2. RNAseq read counts. Table S3. Genes significantly up-regulated by $4 \mathrm{OHT}$ in MOE cells. Table S4. Genes significantly down-regulated by $4 \mathrm{OHT}$ in MOE cells. (DOC 78 kb)

Additional file 2: Figure S1. $E_{2}$ response in putative OVCA precursor cell types. a, b) Densitometry of ERa and PRA protein levels in response to $1 \mathrm{nM} \mathrm{E}_{2}$ treatment (relative to solvent control and normalized to actin) of FVB MOE and FVB MOEp53 ${ }^{\text {R273H }}$ cells. c) qPCR analysis of Pgr induction in response to $24 \mathrm{~h}$ treatment with $1 \mathrm{nM} \mathrm{E}_{2}$ in FVB MOE and FVB MOEp53 ${ }^{\text {R273H }}$ cells. d) FVB MOSE cells treated with an HDAC inhibitor (HD13) does not upregulate ERa or recover $E_{2}$ responsiveness. Significant difference relative to DMSO control denoted by * $(p<0.05)$, ${ }^{* *}(p<0.01)$, and ${ }^{* *}(p<0.001)$. (TIF $\left.150 \mathrm{~kb}\right)$
\end{abstract}

Additional file 3: Figure S2. FVB MOE response to $E_{2}$ and SERMs. $\left.a, b\right)$ qPCR analysis of (a) Pgr and (b) Greb1 induction in response to $48 \mathrm{~h}$ hormone starvation and $24 \mathrm{~h}$ treatment with solvent, $1 \mathrm{nM} \mathrm{E}_{2}$ or $100 \mathrm{nM}$ SERMs. "a" indicates significant difference compared to all treatments. c) Relative growth of FVB MOE following 72-hour hormone starvation and 72-hour treatment with solvent, $1 \mathrm{nM} \mathrm{E}_{2}, 100 \mathrm{nM}$ SERMs and the combination as monitored by SRB assay. d) Relative migration of FVB MOE cells following 72-hour hormone starvation and 24-hour treatment with solvent, $1 \mathrm{nM} \mathrm{E}_{2}$ or $100 \mathrm{nM}$ SERMs. Wound initiated at time of treatment. e) qPCR analysis of Nrip1 expression levels in CD1 and FVB MOE cells in response to $24 \mathrm{~h}$ treatment with DMSO or $1 \mathrm{nM} \mathrm{E}_{2}$. (TIF $615 \mathrm{~kb}$ )

\section{Abbreviation}

DMA: desmethylarzoxifene; $E_{2}$ : Estrogen; ER: estrogen receptor;

FTSEC: fallopian tube secretory epithelial cell; HGSC: high-grade serous ovarian cancer; MOE: murine oviductal epithelium; MOSE: murine ovarian surface epithelium; OSE: ovarian surface epithelium; OVCA: ovarian cancer; PR: progesterone receptor; RAL: raloxifene; SERMs: selective estrogen receptor modulators; $4 \mathrm{OHT}$ : 4-hydroxytamoxifen.

\section{Competing interests}

None.

\section{Authors' contributions}

$\mathrm{GMH}$ and JEB conceived of experiments. GMH, MD, and DAD performed experiments. MJS analyzed RNAseq data. Finally, GMH, MD, and JEB prepared manuscript. All authors approved the final manuscript.

\section{Acknowledgements}

We are grateful to Barbara Vanderhyden for FVB MTEC and MOSE cell lines, Ronny Drapkin for the FTSEC cell line, Greg Thatcher for the SERMs and Pavel Petukov for the HDAC inhibitor. This work was supported by the Northwestern University NGS Core Facility.

\section{Funding}

Funding for J.B. was provided by the American Cancer Society (ACS-RSG-12230-01); the National Center for Clinical and Translational Science, Office for Research in Women's Health, and the National Institute for Environmental and Health Safety through grant UH3TR001207, and the National Institutes of Health (R01EB014806). This work was funded by the Chicago Biomedical Consortium with support from the Searle Funds at The Chicago Community Trust (G.MH).

\section{Author details}

${ }^{1}$ Department of Medicinal Chemistry and Pharmacognosy, University of Illinois at Chicago, Chicago, Illinois 60607, USA. ${ }^{2}$ Department of Human Biology, University of Wisconsin - Green Bay, Green Bay, Wisconsin 54311, 
USA. ${ }^{3}$ Next Generation Sequencing Core Facility, Feinberg School of Medicine, Northwestern University, Chicago, Illinois 60611, USA.

\section{Received: 12 November 2015 Accepted: 3 February 2016 Published online: 15 February 2016}

\section{References}

1. DeSantis CE, Lin CC, Mariotto AB, Siegel RL, Stein KD, Kramer JL, et al. Cancer treatment and survivorship statistics, 2014. CA Cancer J Clin. 2014;64:252-71.

2. Li J, Fadare O, Xiang L, Kong B, Zheng W. Ovarian serous carcinoma: recent concepts on its origin and carcinogenesis. J Hematol Oncol. 2012;5:8.

3. Bell DA. Origins and molecular pathology of ovarian cancer. Mod Pathol. 2005;18:S19-32.

4. Marquez RT, Baggerly KA, Patterson AP, Liu J, Broaddus R, Frumovitz M, et al. Patterns of gene expression in different histotypes of epithelial ovarian cancer correlate with those in normal fallopian tube, endometrium, and colon. Clin Cancer Res. 2005;11:6116-26.

5. Lee Y, Miron A, Drapkin R, Nucci MR, Medeiros F, Saleemuddin A, et al. A candidate precursor to serous carcinoma that originates in the distal fallopian tube. J Pathol. 2007;211:26-35

6. Kuhn E, Kurman RJ, Shih IM. Ovarian Cancer Is an Imported Disease: Fact or Fiction? Curr Obstet Gynecol Rep. 2012;1:1-9.

7. Perets R, Wyant GA, Muto KW, Bijron JG, Poole BB, Chin KT, et al. Transformation of the fallopian tube secretory epithelium leads to highgrade serous ovarian cancer in Brca;Tp53:Pten models. Cancer Cell. 2013:24:751-65

8. Lacey Jr JV, Mink PJ, Lubin JH, Sherman ME, Troisi R, Hartge P, et al. Menopausal hormone replacement therapy and risk of ovarian cancer JAMA. 2002;288:334-41.

9. Menezo Y, Guerin P. The mammalian oviduct: biochemistry and physiology. Eur J Obstet Gynecol Reprod Biol. 1997;73:99-104.

10. Katzenellenbogen BS, Choi I, Delage-Mourroux R, Ediger TR, Martini PG, Montano $\mathrm{M}$, et al. Molecular mechanisms of estrogen action: selective ligands and receptor pharmacology. J Steroid Biochem Mol Biol. 2000;74:279-85

11. Prossnitz ER, Barton M. The G-protein-coupled estrogen receptor GPER in health and disease. Nat Rev Endocrinol. 2011;7:715-26.

12. Collaborative Group On Epidemiological Studies Of Ovarian C. Menopausal hormone use and ovarian cancer risk: individual participant meta-analysis of 52 epidemiological studies. Lancet. 2015;385:1835-42.

13. Sieh W, Kobel M, Longacre TA, Bowtell DD, deFazio A, Goodman MT, et al. Hormone-receptor expression and ovarian cancer survival: an Ovarian Tumor Tissue Analysis consortium study. Lancet Oncol. 2013;14:853-62.

14. Sjoquist KM, Martyn J, Edmondson RJ, Friedlander ML. The role of hormonal therapy in gynecological cancers-current status and future directions. Int J Gynecol Cancer. 2011;21:1328-33.

15. Cheskis BJ, Greger JG, Nagpal S, Freedman LP. Signaling by estrogens. J Cell Physiol. 2007;213:610-7.

16. Quartuccio SM, Lantvit DD, Bosland MC, Burdette JE. Conditional inactivation of p53 in mouse ovarian surface epithelium does not alter MIS driven Smad2-dominant negative epithelium-lined inclusion cysts or teratomas. PLoS One. 2013:8:e65067.

17. Quartuccio SM, Karthikeyan S, Eddie SL, Lantvit DD, Ó hAinmhire E, Modi DA, Wei JJ, Burdette JE. Mutant p53 expression in fallopian tube epithelium drives cell migration. International Journal of Cancer 2015; In Press.

18. Green B, Leake, RE. Steroid Hormones: A practical approach, IRL Press, Oxford, UK, 1987.

19. Skehan P, Storeng R, Scudiero D, Monks A, McMahon J, Vistica D, et al. New colorimetric cytotoxicity assay for anticancer-drug screening. J Natl Cancer Inst. 1990;82:1107-12.

20. King SM, Hilliard TS, Wu LY, Jaffe RC, Fazleabas AT, Burdette JE. The impact of ovulation on fallopian tube epithelial cells: evaluating three hypotheses connecting ovulation and serous ovarian cancer. Endocr Relat Cancer. 2011;18:627-42

21. Townsend TR, Moyle-Heyrman G, Sukerkar PA, MacRenaris KW, Burdette JE, Meade TJ. Progesterone-targeted magnetic resonance imaging probes. Bioconjug Chem. 2014;25:1428-37.

22. Trapnell C, Roberts A, Goff L, Pertea G, Kim D, Kelley DR, et al. Differentia gene and transcript expression analysis of RNA-seq experiments with TopHat and Cufflinks. Nat Protoc. 2012;7:562-78.
23. Tabas-Madrid D, Nogales-Cadenas R, Pascual-Montano A. GeneCodis3: a non-redundant and modular enrichment analysis tool for functional genomics. Nucleic Acids Res. 2012;40:W478-83.

24. Nogales-Cadenas R, Carmona-Saez P, Vazquez M, Vicente C, Yang X, Tirado $F$, et al. GeneCodis: interpreting gene lists through enrichment analysis and integration of diverse biological information. Nucleic Acids Res. 2009:37:W317-22

25. Carmona-Saez P, Chagoyen M, Tirado F, Carazo JM, Pascual-Montano A GENECODIS: a web-based tool for finding significant concurrent annotations in gene lists. Genome Biol. 2007;8:R3.

26. Alarid ET, Bakopoulos N, Solodin N. Proteasome-mediated proteolysis of estrogen receptor: a novel component in autologous down-regulation. Mol Endocrinol. 1999:13:1522-34.

27. Cancer Genome Atlas Research N. Integrated genomic analyses of ovarian carcinoma. Nature. 2011:474:609-15.

28. Karst AM, Levanon K, Drapkin R. Modeling high-grade serous ovarian carcinogenesis from the fallopian tube. Proc Natl Acad Sci U S A. 2011;108:7547-52

29. Bai W, Oliveros-Saunders B, Wang Q, Acevedo-Duncan ME, Nicosia SV. Estrogen stimulation of ovarian surface epithelial cell proliferation. In Vitro Cell Dev Biol Anim. 2000;36:657-66.

30. Patel HK, Siklos MI, Abdelkarim H, Mendonca EL, Vaidya A, Petukhov PA, et al. A chimeric SERM-histone deacetylase inhibitor approach to breast cancer therapy. Chem Med Chem. 2014;9:602-13.

31. Advani P, Moreno-Aspitia A. Current strategies for the prevention of breast cancer. Breast Cancer (Dove Med Press). 2014;6:59-71.

32. O'Donnell AJ, Macleod KG, Burns DJ, Smyth JF, Langdon SP. Estrogen receptor-alpha mediates gene expression changes and growth response in ovarian cancer cells exposed to estrogen. Endocr Relat Cancer. 2005;12:851-66.

33. Spillman MA, Manning NG, Dye WW, Sartorius CA, Post MD, Harrell JC, et al. Tissue-specific pathways for estrogen regulation of ovarian cancer growth and metastasis. Cancer Res. 2010;70:8927-36.

34. Croxatto HB. Physiology of gamete and embryo transport through the fallopian tube. Reprod Biomed Online. 2002;4:160-9.

35. Augereau P, Badia E, Fuentes M, Rabenoelina F, Corniou M, Deroca D, et al. Transcriptional regulation of the human NRIP1/RIP140 gene by estrogen is modulated by dioxin signalling. Mol Pharmacol. 2006;69:1338-46.

36. Umezu T, Tomooka Y. An evidence of stromal cell populations functionally linked with epithelial cell populations in the mouse oviduct. Zoolog Sci. 2004:21:319-26.

37. Domcke S, Sinha R, Levine DA, Sander C, Schultz N. Evaluating cell lines as tumour models by comparison of genomic profiles. Nat Commun. 2013;4:2126.

38. Lau KM, Mok SC, Ho SM. Expression of human estrogen receptor-alpha and -beta, progesterone receptor, and androgen receptor mRNA in normal and malignant ovarian epithelial cells. Proc Natl Acad Sci U S A. 1999:96:5722-7.

39. Li Y, Arao Y, Hall JM, Burkett S, Liu L, Gerrish K, et al. Research Resource: STR DNA profile and gene expression comparisons of human BG-1 cells and a BG-1/MCF-7 clonal variant. Mol Endocrinol. 2014;28:2072-81.

40. Eddie SL, Quartuccio SM, Zhu J, Shepherd JA, Kothari R, Kim JJ, et al. Threedimensional modeling of the human fallopian tube fimbriae. Gynecol Oncol. 2015:136:348-54.

41. Chene G, Radosevic-Robin N, Tardieu AS, Cayre A, Raoelfils I, Dechelotte P, et al. Morphological and immunohistochemical study of ovarian and tubal dysplasia associated with tamoxifen. Eur J Histochem. 2014;58:2251.

42. Liu X, Pisha E, Tonetti DA, Yao D, Li Y, Yao J, et al. Antiestrogenic and DNA damaging effects induced by tamoxifen and toremifene metabolites. Chem Res Toxicol. 2003;16:832-7.

43. Ghosh MG, Thompson DA, Weigel RJ. PDZK1 and GREB1 are estrogenregulated genes expressed in hormone-responsive breast cancer. Cancer Res. 2000;60:6367-75.

44. Robertson SA, Mayrhofer G, Seamark RF. Ovarian steroid hormones regulate granulocyte-macrophage colony-stimulating factor synthesis by uterine epithelial cells in the mouse. Biol Reprod. 1996;54:183-96.

45. Welboren WJ, Sweep FC, Span PN, Stunnenberg HG. Genomic actions of estrogen receptor alpha: what are the targets and how are they regulated? Endocr Relat Cancer. 2009:16:1073-89.

46. Mulero JJ, Boyle BJ, Bradley S, Bright JM, Nelken ST, Ho TT, et al. Three new human members of the lipid transfer/lipopolysaccharide binding protein family (LT/LBP). Immunogenetics. 2002;54:293-300. 
47. Huber-Keener K, Liu X, Wang Z, Wang Y, Freeman W, Wu S, et al. Differential gene expression in tamoxifen-resistant breast cancer cells revealed by a new analytical model of RNA-Seq data. PLoS One. 2012:7:e41333.

48. Breitbart $\mathrm{H}$. Intracellular calcium regulation in sperm capacitation and acrosomal reaction. Mol Cell Endocrinol. 2002;187:139-44.

49. Lyons RA, Saridogan E, Djahanbakhch O. The reproductive significance of human Fallopian tube cilia. Hum Reprod Update. 2006;12:363-72.

50. Cerami E, Gao J, Dogrusoz U, Gross BE, Sumer SO, Aksoy BA, et al. The cBio cancer genomics portal: an open platform for exploring multidimensional cancer genomics data. Cancer Discov. 2012;2:401-4.

51. Gao J, Aksoy BA, Dogrusoz U, Dresdner G, Gross B, Sumer SO, et al. Integrative analysis of complex cancer genomics and clinical profiles using the cBioPortal. Sci Signal. 2013;6:pl1.

52. Lee JY, Jeong W, Kim JH, Kim J, Bazer FW, Han JY, et al. Distinct expression pattern and post-transcriptional regulation of cell cycle genes in the glandular epithelia of avian ovarian carcinomas. PLoS One. 2012;7:e51592.

53. Sakuma K, Aoki M, Kannagi R. Transcription factors c-Myc and CDX2 mediate E-selectin ligand expression in colon cancer cells undergoing EGF/ bFGF-induced epithelial-mesenchymal transition. Proc Natl Acad Sci U S A. 2012;109:7776-81.

54. Noel EE, Yeste-Velasco M, Mao X, Perry J, Kudahetti SC, Li NF, et al. The association of CCND1 overexpression and cisplatin resistance in testicular germ cell tumors and other cancers. Am J Pathol. 2010;176:2607-15.

55. Beaufort CM, Helmijr JC, Piskorz AM, Hoogstraat M, Ruigrok-Ritstier K, Besselink N, et al. Ovarian cancer cell line panel (OCCP): clinical importance of in vitro morphological subtypes. PLoS One. 2014;9:e103988.

\section{Submit your next manuscript to BioMed Central and we will help you at every step:}

- We accept pre-submission inquiries

- Our selector tool helps you to find the most relevant journal

- We provide round the clock customer support

- Convenient online submission

- Thorough peer review

- Inclusion in PubMed and all major indexing services

- Maximum visibility for your research

Submit your manuscript at www.biomedcentral.com/submit
Biomed Central 